\title{
When the Largest Spiral is Formed
}

\author{
Rafael T. Eufrasio ${ }^{1,2}$, Duília F. de Mello ${ }^{1,2,3}$, Fernanda \\ Urrutia-Viscarra $^{4}$, Claudia Mendes de Oliveira ${ }^{4}$, and Eli Dwek ${ }^{1}$ \\ ${ }^{1}$ The Catholic University of America, Washington, DC 20064, USA \\ ${ }^{2}$ NASA Goddard Space Flight Center, Greenbelt, MD 20771, USA \\ ${ }^{3}$ Johns Hopkins University, Baltimore, MD 21218, USA \\ ${ }^{4}$ Universidade de São Paulo. São Paulo, SP 05508-090, Brazil
}

\begin{abstract}
We used UV-to-IR archival data to investigate the nature of the giant spiral galaxy, NGC 6872, 65 Mpc away. It belongs to the southern Pavo group and is interacting with a small lenticular galaxy, IC4970. GALEX UV images show a very large part of the galaxy not seen before, making it one of the largest spiral galaxies known, with a physical size greater than $150 \mathrm{kpc}$. The SED of 17 regions ( $10 \mathrm{kpc}$ of diameter) across the two arms show a remarkable spatial distribution, as if they were mirror images with respect to their stellar population. However, the last $40 \mathrm{kpc}$ of the northeastern arm are much bluer than any other region of the southwestern one. There is a strong spatial correlation between the NUV luminosity and the distance to the nucleus. The UV data supports the scenario of an interaction at $130 \mathrm{Myr}$ which triggered star formation all over the disk of NGC 6872. The tip of the northeastern arm resembles a tidal dwarf galaxy in the process of formation.
\end{abstract}

Keywords. galaxies: spiral — galaxies: stellar content — galaxies: structure

We used UV-to-IR photometric data taken with GALEX (FUV and NUV), VLT $(U B V R I), 2 \mathrm{MASS}\left(J H K_{s}\right)$, and Spitzer (IRAC) to analyze the properties of the giant spiral NGC 6872. We have convolved all the images to the same 5.3" FHWM spatial resolution, reprojected them to the same astrometric grid (pixel size of $1.0^{\prime \prime}$ ) and obtained the SED for each pixel. We performed photometry across the galaxy in circular apertures with diameters of $32^{\prime \prime}$ (or $10 \mathrm{kpc}$ at $65 \mathrm{Mpc}$ ). When the SEDs are normalized by the $4.5 \mu \mathrm{m}$ fluxes, the FUV and NUV fluxes span a wide range of a thousand, while the main properties of the normalized SEDs are almost unchanged from the $R$ band to $4.5 \mu \mathrm{m}$. We also find an intriguing symmetry between regions in the southwestern and the northeastern arms having nearly identical SEDs. There is also a strong UV source at the tip of the northeastern arm resembling a tidal dwarf galaxy. This source is 90 kpc away from the nucleus and if we consider it as part of NGC 6872, the whole galaxy measures $160 \mathrm{kpc}$. As proposed by numerical simulations (Mihos et al. 1993 and Horellou \& Koribalski 2007), NGC 6872 suffered a major collision with IC 4970 around 130 Myr ago. Our results agree with a global mixing of the pre-collision stellar population all over the disk of NGC 6872 at that epoch and a consequent star formation propagation to the outskirts of the galaxy. The mixing may have contributed to triggering star formation, in agreement with what was predicted by Bastian et al. (2005). The last $40 \mathrm{kpc}$ of the northeastern arm is UV-brighter than any region in the other arm, having the youngest population and least contamination by pre-collision stars thrown there by the encounter.

\section{References}

Bastian, N., Hempel, M., Kissler-Patig, M., Homeier, N. L., \& Trancho, G. 2005, A\&A A, 435, 65 Horellou, C. \& Koribalski, B. 2007, A\&A A, 464, 155

Mihos, J. C., Bothun, G. D., \& Richstone, D. O. 1993, ApJ, 418, 82 